\title{
冷轧不锈钢丝网增强铝基复合板性能分析"
}

\author{
黄华贵 王计超 刘文文 杜凤山 \\ (燕山大学国家冷轧板带装备及工艺工程技术研究中心 秦皇岛 066004)
}

\begin{abstract}
摘要：以冷轧复合法制备不锈钢丝网增强铝基复合板, 采用金相显微镜观察复合界面微观形貌随压下率变化的演变规律, 利 用拉伸试验机获得复合板力学性能。结果表明: 随着压下率增大, 复合界面由最初的钢丝网与铝板间机械咬合转变为钢丝网 交叉点与铝板间的局部点焊合，随后为钢丝网交叉点与铝板间的面焊合及非交叉点处钢丝与铝板间的局部点焊合; 当压下率 达到 52.8\%时, 钢丝网与铝板间形成全面焊合及铝板与铝板间的结合。复合板力学性能测试结果显示: $0^{\circ}$ 钢丝网增强复合板 抗拉强度、比抗拉强度在一定范围内随着冷轧压下率、钢丝网目数的增大而提高, 随铝板初始厚度增大呈下降趋势, 当铝板 初始厚度大于 $2 \mathrm{~mm}$ 后, 钢丝网增强效果较小。不同摆放角度钢丝网增强复合板较无钢丝网增强铝-铝复合板抗拉强度有所提 高, $0^{\circ}$ 钢丝网增强复合板提高 $22.5 \%, 45$ 钢丝网增强复合板提高 $34.2 \%$, 但伸长率都有所下降, 其中 45 钢丝网增强复合板 伸长率下降较少。
\end{abstract}

关键词: 不锈钢丝网增强铝基复合板; 冷轧; 抗拉强度; 复合界面; 比抗拉强度

中图分类号: TG339

\section{Analysis on Mechanical Properties of Stainless Steel Wire Mesh Reinforced Aluminum Composite Plate Fabricated by Cold Rolling}

\author{
HUANG Huagui WANG Jichao LIU Wenwen DU Fengshan
}

(National Engineering Research Center for Equipment and Technology of Cold Strip Rolling,

Yanshan University, Qinhuangdao 066004)

\begin{abstract}
Aiming at the stainless steel wire mesh reinforced aluminium composite plate fabricated by cold rolling process, the evolution of the microstructure of the composite interface with reduction ratio changing is observed by metallographic microscope, and the mechanical properties of the composite plate is investigated by tensile testing machine. The results show that with the increasing of reduction ratio, the boning interface changes from the original mechanical occluding between wire mesh and aluminium plate to the local point welding between wire mesh’s overlapping point and aluminium plate, then it turns to the surface welding of wire mesh's overlapping point and aluminium plate as well as the local point welding of not overlapping point area's wire and aluminium plate. When the reduction ratio reaches $52.8 \%$, comprehensive welding is formed between wire mesh and aluminium plate as well as the bonding between aluminium plate and aluminium plate. The test results of composite plate' s mechanical performance show that in a certain range, the tensile strength and specific tensile strength of the composite plate increase with the increasing of reduction ratio and number of wire mesh, and both of them show a downward trend with the increasing of the initial thickness of aluminium plate. Steel wire mesh reinforcement effect is small when the aluminium plate's initial thickness is more than 2 mm. Compared to the unreinforced $\mathrm{Al} / \mathrm{Al}$ composite plate, tensile strength of the composite plate reinforced with steel wire mesh placed at different orientations is higher. The tensile strength of $0^{\circ}$ steel wire reinforced composite plate increases by $22.5 \%$ and the tensile strength of $45^{\circ}$ steel wire reinforced composite plate increases by $34.2 \%$. But percent elongation is lower, and percent elongation of $45^{\circ}$ steel wire reinforced composite plate decreases less.
\end{abstract}

Key words: stainless steel wire mesh reinforced aluminium composite plate; cold rolling; tensile strength; composite interface; specific tensile strength

* 国家自然科学基金(51474189)和河北省高等学校科学技术研究 (QN2015214)资助项目。20161227 收到初稿, 20170621 收到修改稿 


\section{0 前言}

航空航天、海洋工程、轨道交通等国家战略新 兴产业的快速发展, 对材料性能的要求也日益提高, 单一组元材料已经很难满足需求, 研制新型复合材 料成为材料科学与工程领域的重要研究方向 ${ }^{[1-3]}$ 。铝 及铝合金属于轻质合金, 具有密度小、比强度高、 比刚度高、抗疲劳性能好、耐磨损性能优异等优点, 成为应用最广泛的金属材料之一[4-5]。为了进一步提 高铝合金材料的综合性能, 国内外学者通过将碳纤 维、嗍纤维、玻璃纤维、 $\mathrm{SiC}$ 颗粒、石墨颗粒、 $\mathrm{BN}$ 颗粒等添加到铝合金中, 提出了多种新型铝基复合 材料制备工艺 ${ }^{[6-9]}$ 。上述增强相能够提升铝合金的一 些性能, 但是由于大部分与铝的润湿性差, 而且制 备成本过高, 主要应用于军事等特殊领域。

为了获得低价的新型铝基复合材料, 众多学者将 目光放到了与铝润湿性好、成本低廉的钢丝及钢丝网 上, 并进行了大量的试验研究。其中, HAGA $\mathrm{T}$ 等利 用 “双辊铸轧法” 制备出了界面结合良好、无孔洞的 钢丝增强铝基复合板，但没有报道其力学性能 ${ }^{[10-11]}$ 。 日本学者采用分流模挤压复合法制备钢丝增强 6063 铝合金管, 将铝合金管的抗拉强度、抗弯强度及压缩 失稳强度均提高 $45 \%$ ～ $50 \%$, 而重量增加不到 $15 \%{ }^{[12]}$ 。 PAKZAMAN 等 ${ }^{[13]}$ 通过消失模铸造法制备了钢丝网增 强铝基复合材料, 比较了钢丝网有无镀镍涂层对复合 板结合及力学性能的影响, 表明有镀镍涂层的钢丝网 增强复合材料性能较好。GÜLENÇ 等 ${ }^{[14]}$ 利用爆炸复合 法制备了钢丝网增强铝基复合板，使其抗拉强度提高 了 20\% 30\%。SZCZEPANIK 等 ${ }^{[15]}$ 通过闭式热模锻法 制备了钢丝网增强铝基复合材料, 测得其弯曲强度达 431 478 MPa。

综上，钢丝网作为铝基复合材料的增强相可以 提升整体性能已经被学者所证实。然而, 钢丝网增 强铝基复合材料的制备工艺还非常单一，研究不同 工艺下钢丝网增强铝基复合材料结合过程及材料性 能具有重要意义。为此, 鉴于轧制在板带复合中的 广泛应用, 本文采用冷轧工艺制备不锈钢丝网增强 铝基复合板, 探究钢丝网与铝基体界面结合过程和 界面复合条件, 分析轧制参数、组坏方式对钢丝网 增强铝基复合板力学性能的影响规律。

\section{1 冷轧复合试验}

为验证冷轧复合工艺可行性, 本文基体材料选 择塑性较好的 1060 铝板, 增强相选择 $0.5 \mathrm{~mm}$ 丝径
304 钢丝网, 铝板和钢丝网尺寸均为 $100 \mathrm{~mm} \times 400$ $\mathrm{mm}$ 。为去除毛坏表面氧化膜和油污, 铝板表面采用 $10 \% \mathrm{HCl}$ 溶液酸洗，再用钢丝刷打磨、丙酮浸泡、 酒精清洗后自然风干; 钢丝网表面采用 $10 \% \mathrm{NaOH}$ 溶液碱洗, 再用丙酩浸泡、酒精清洗后自然风干。 将两块等厚的铝板与一块钢丝网按照 “ $\mathrm{Al} / 304$ 钢丝 网/Al” 方式叠置后, 在 $\phi 240 \mathrm{~mm} \times 200 \mathrm{~mm}$ 二辊轧机 上进行轧制(图 1), 轧制速度为 $2 \mathrm{~m} / \mathrm{min}$, 试验工况 如表 1 所示, 钢丝网布置方式如图 2 所示。并定义 复合板轧制压下率 $\eta$ 如式(1)所示

$$
\eta=\left(h+h_{w}-h_{0}\right) /\left(h+h_{w}\right)
$$

式中, $h$ 为铝板厚度; $h_{w}$ 为钢丝网厚度; $h_{0}$ 为轧后 复合板厚度。为对比复合板增强效果, 取 2 块相同 1060 铝板冷轧到与工况 5、11 复合板同等厚度, 制 取无钢丝网增强的铝-铝复合板。

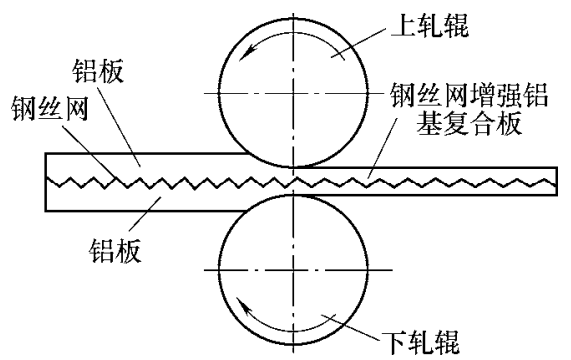

图 1 轧制示意图

表 1 轧制工艺表

\begin{tabular}{ccccc}
\hline 工况 & $\begin{array}{c}\text { 压下率 } \eta \\
(\%)\end{array}$ & $\begin{array}{c}\text { 钢丝网 } \\
\text { 目数 } \alpha\end{array}$ & $\begin{array}{c}\text { 钢丝网 } \\
\text { 摆放角度 } \theta /\left(^{\circ}\right)\end{array}$ & $\begin{array}{c}\text { 铝板厚度 } \\
h / \mathrm{mm}\end{array}$ \\
\hline 工况 1 & 24.8 & 12 & 0 & 0.9 \\
工况 2 & 32.8 & 12 & 0 & 0.9 \\
工况 3 & 46.4 & 12 & 0 & 0.9 \\
工况 4 & 51.2 & 12 & 0 & 0.9 \\
工况 5 & 52.8 & 12 & 0 & 0.9 \\
工况 6 & 46.4 & 8 & 0 & 0.9 \\
工况 7 & 46.4 & 20 & 0 & 0.9 \\
工况 8 & 46.4 & 20 & 0 & 2.3 \\
工况 9 & 46.4 & 20 & 0 & 3.3 \\
工况 10 & 46.4 & 20 & 0 & 4.4 \\
工况 11 & 52.8 & 12 & 45 & 0.9 \\
\hline
\end{tabular}

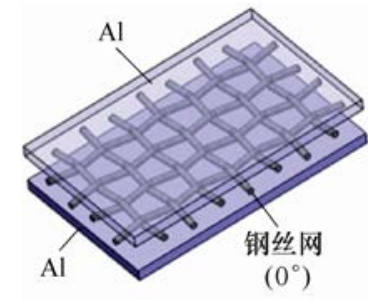

(a) 钢丝网 $0^{\circ}$ 摆放

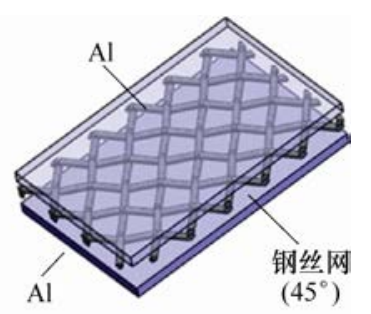

(b) 钢丝网 $45^{\circ}$ 摆放
图 2 钢丝网布置方式示意图

由于钢丝网编织特点, 在轧制中会出现钢丝交 叉点与铝板复合界面、非交叉点处钢丝与铝板复合 
界面以及铝板和铝板复合界面(图 3), 利用 Axiovert 200MAT 光学显微镜观察不同复合界面的演变过程。
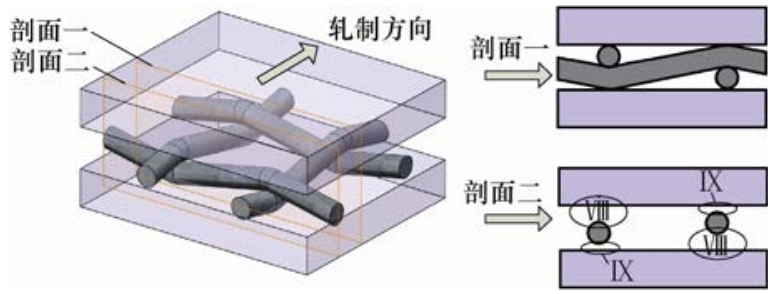

图 3 复合板剖切位置示意图

为了获得复合板的力学性能, 按照图 4 所示尺 寸制备复合板的拉伸试件, 利用 INSPEKT TABLE 100 电子万能材料试验机进行拉伸试验研究, 拉伸 速率为 $1 \mathrm{~mm} / \mathrm{min}$, 测量其抗拉强度 $\sigma_{\mathrm{b}}$ 。并采用天 平量筒法测量复合板密度 $\rho$ 。由于钢丝网加入会提 升板坏质量, 定义比抗拉强度 $\sigma_{\mathrm{bk}}$ 用来表征材料的 力学性能。计算公式为: $\sigma_{\mathrm{bk}}=\sigma_{\mathrm{b}} / \rho$ 。

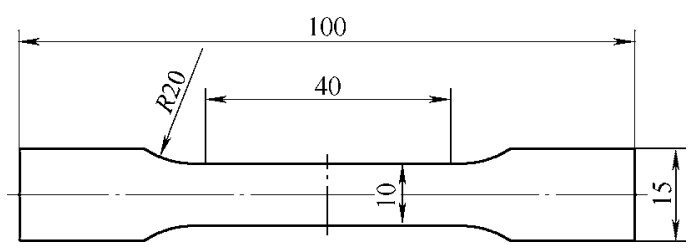

图 4 拉伸试件

\section{2 结果与分析}

\section{1 界面结合过程}

图 5 为不同压下率 $\eta$ 下钢丝网交叉点与铝板复 合界面(图 3 中剖面一)。当压下率为 32.8\%时，I 区 内钢丝与铝板之间没有明显的孔隙出现, 界面结合 良好; II 区内钢丝与铝板之间存在连续的孔隙，特 别是在交叉点处有较大的孔洞存在, 界面结合较差; III 区内钢丝与铝板之间存在间断式孔隙, 界面实现 初步结合，但结合效果一般。复合界面出现不同结 合情况是由于钢丝网编织的特点, 在轧制过程中 I 区、III 区内钢丝先与铝板接触结合，II 区内钢丝需 要较大的变形量才能与铝板接触, 从而 I 区、III 区 内钢丝与铝板的结合优于 II 区。同时在 I 区内铝板 在轧制力作用下会包裹钢丝，铝板与钢丝接触面会 被拉长露出更多新鲜金属, 更易实现界面结合, 从 而 I 区钢丝与铝板的结合优于 III 区。当压下率为 $46.4 \%$ 时，钢丝网交叉点与铝板之间已经不存在明 显的孔隙, 界面结合良好。但依然存在小部分残余 孔隙(图 b 中 V 区)。当压下率为 $52.8 \%$ 时, 铝板与钢 丝网结合良好，无残余孔隙出现。

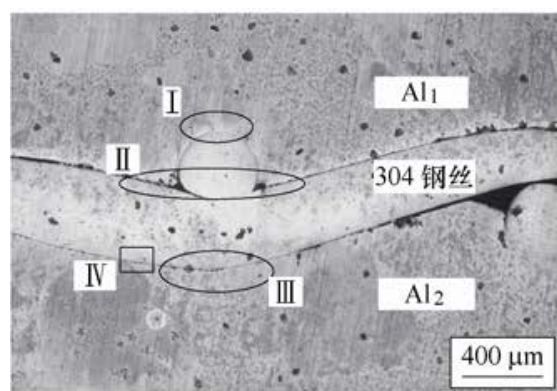

(a) $\eta=32.8 \%$

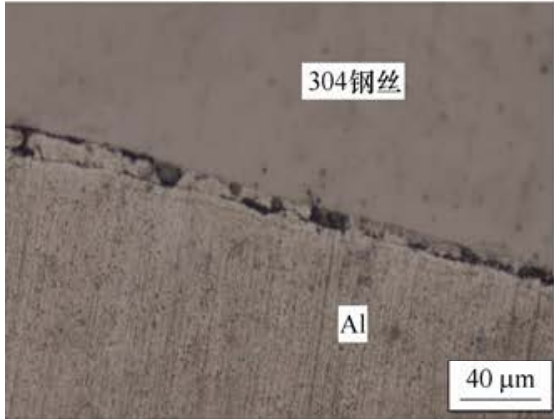

(d) IV放大图

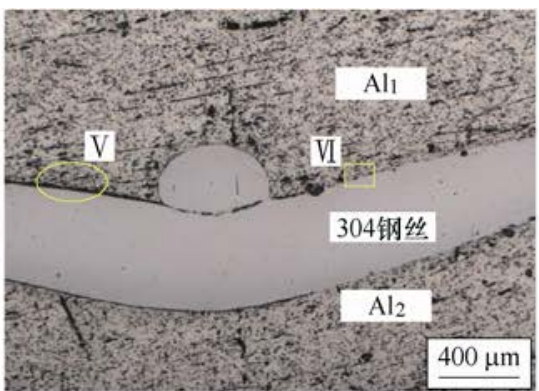

(b) $\eta=46.4 \%$

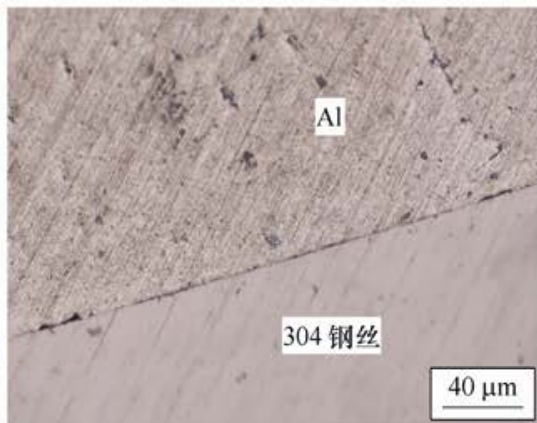

(e) VI放大图

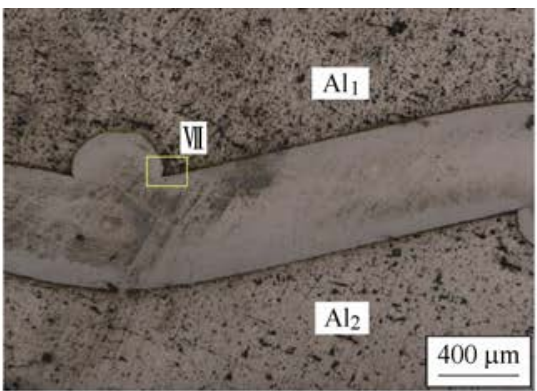

(c) $\eta=52.8 \%$

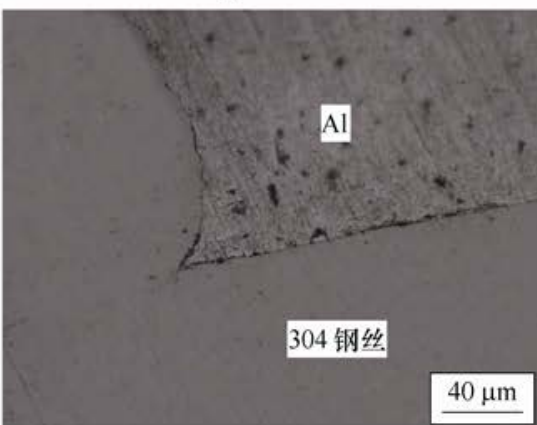

(f) UI放大图

图 5 不同压下率 $\eta$ 下钢丝网交叉点与铝板复合界面

图 6 为不同压下率 $\eta$ 下非交叉点处钢丝与铝板 复合界面(图 3 中剖面二)。当压下率为 32.8\%时, 图 6a 中VIII区内钢丝与铝板的结合情况明显优于IX区。 VIII 区内钢丝与铝板间存在部分连续残余孔隙, 实现
局部结合, IX区内钢丝与铝板间存在较大缝隙，没 有实现结合, 究其原因, 由图 3 所示, 复合板在冷 轧过程中，IX区内钢丝与铝板接触所需压下率要大 于VII区。 $\mathrm{Al}_{1} 、 \mathrm{Al}_{2}$ 之间存在明显连续的缝隙, 界面 
并未结合。当压下率为 46.4\%时, 图 $6 \mathrm{~b}$ 中VIII区内钢 丝与铝板间基本不存在残余孔隙, 结合较好, VIII区 内钢丝与铝板间缝隙变小但依然没有实现结合。 $\mathrm{Al}_{1} 、 \mathrm{Al}_{2}$ 间缝隙变小, 界面仍未结合。当压下率为 $51.2 \%$ 时, 钢丝与铝板间无明显残余孔隙, 钢丝与 $\mathrm{Al}_{1} 、 \mathrm{Al}_{2}$ 间结合差距已不明显, 都结合较好。 $\mathrm{Al}_{1}$ 、 $\mathrm{Al}_{2}$ 间存在不连续的残余孔隙, 界面实现局部结合。 当压下率为 $52.8 \%$ 时, 钢丝与铝板间界面在放大 500 倍下依然无残余孔隙, 结合良好。 $\mathrm{Al}_{1} 、 \mathrm{Al}_{2}$ 间同样 无残余孔隙, 界面实现良好结合。

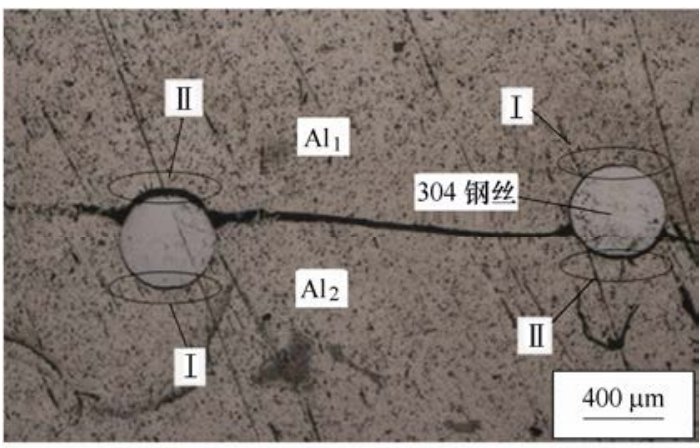

(a) $\eta=32.8 \%$

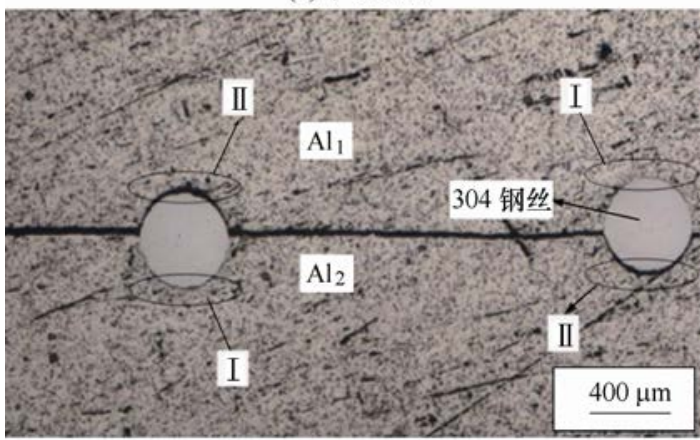

(b) $\eta=46.4 \%$

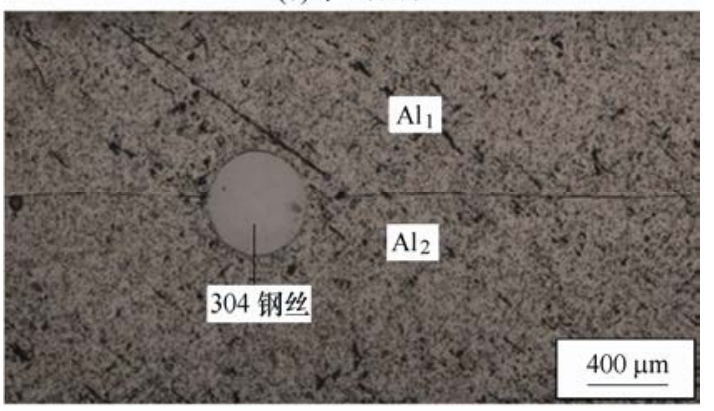

(c) $\eta=51.2 \%$

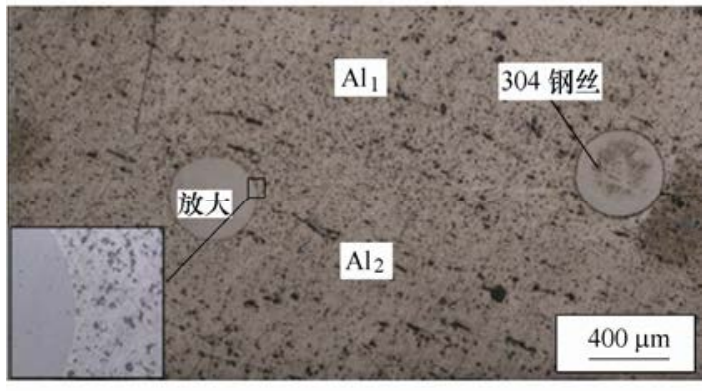

(d) $\eta=52.8 \%$

图 6 不同压下率 $\eta$ 下非交叉点处钢丝与铝板复合界面
比较压下率为 $46.4 \%$ 时三种复合界面的结合情 况, 可以看出钢丝网交叉点与铝板之间界面结合良 好, 非交叉点处钢丝与铝板之间为局部结合良好, $\mathrm{Al}_{1} 、 \mathrm{Al}_{2}$ 间依然存在明显的缝隙。这是由于钢丝网 的编织特点导致复合板在冷轧过程中钢丝网交叉点 先与铝板接触, 后是非交叉点处钢丝与铝板接触, 而上下铝板间未实现接触, 从而在相同的压下率下, 钢丝网交叉点与铝板的结合更好。

综合图 5、图 6 总体来讲，压下率较小时硬质 钢丝网在轧制力作用下嵌入软质铝基体中，复合板 实现机械咬合。随着轧制压下率的增大，压下率在 $32.8 \%$ 时, 钢丝网交叉点与铝板实现局部点焊合, 此时在轧制力作用下钢丝网交叉点被铝板包裹, 铝 板被拉长而发生相对于在同等压下率下钢板-铝板 冷轧复合更大的变形, 率先实现界面的结合。随着 轧制压下率的继续增大，复合界面处更多暴露出来 的纯金属原子被激活，焊合点密度变大并开始向面 焊合演变，压下率在 $46.4 \%$ 时，钢丝网交叉点与铝 板界面出现了较大的面焊合，结合强度得到较大提 高, 已很难撕裂。当压下率达到 52.8\%时, 钢丝网 与铝板界面达到全面焊合，同时 $\mathrm{Al}_{1} 、 \mathrm{Al}_{2}$ 也得到充 分接触并结合。

\section{2 力学性能分析}

\subsection{1 轧制参数的影响}

不同压下率 $\eta$ 下(工况 1 3、5)复合板抗拉强度、 比抗拉强度如图 7 所示。从图中可以看出，随着压 下率的增大，复合板抗拉强度、比抗拉强度增大。 不同压下率 $\eta$ 下复合板拉伸试样拉断后侧视形貌如 图 8 所示。可以看出当压下率为 $24.8 \%$ 时, 复合板 结合强度较差, 在拉伸过程中钢丝网不断裂, 铝板 被拉长并断裂, 钢丝网基本起不到增强效果, 复合 板抗拉强度较低。随着压下率的增大, 在压下率为 $32.8 \%$ 时铝板与钢丝网之间实现局部结合, 钢丝网 作为增强相能够对复合板抗拉强度做出贡献, 抗拉 强度增加。当压下率为 $46.4 \%$ 时, 钢丝网能够较充 分地发挥作用, 在拉伸过程中钢丝网发生断裂, 抗 拉强度继续增强。随后压下率进一步增大，复合板 抗拉强度进一步提高。

\subsection{2 组坏方式的影响}

(1) 钢丝网目数的影响。不同目数 $\alpha$ (工况 3、6、 7)的复合板抗拉强度、比抗拉强度如图 9 所示。从 图中可以看出, 随着钢丝网目数的增加, 复合板抗 拉强度、比抗拉强度增加。钢丝网目数增加, 钢丝 网在单位体积中所占的比例增大, 从而使复合板抗 拉强度提高。

(2) 铝板初始厚度的影响。不同铝板初始厚度 
$h($ 工况 3、8、9、10)的复合板抗拉强度、比抗拉强 度如图 10 所示。从图中可以看出, 随着铝板厚度增 加, 复合板抗拉强度、比抗拉强度呈下降趋势。铝 板初始厚度增加, 钢丝网丝径不变, 轧制压下率不 变, 从而使复合板单位体积内钢丝网含量减少, 复 合板抗拉强度下降。铝板初始厚度大于 $2 \mathrm{~mm}$ 后, 钢丝网增强效果较小。当采用 $3.32 \mathrm{~mm} 、 4.46 \mathrm{~mm}$ 厚铝板为基材制备钢丝网增强复合板时, 钢丝网沿 轧制方向出现大部分断裂(图 11), 导致钢丝网基本 起不到增强效果。铝板初始厚度增大, 压下率不变, 压下量增大, 当压下量太大时引起的轧件纵向伸长 率超过钢丝网自身延展率时, 导致钢丝网沿轧制方 向断裂, 钢丝网起不到增强作用。同样铝板初始厚 度不变, 冷轧压下率太大也会出现钢丝网断裂, 起 不到增强效果。

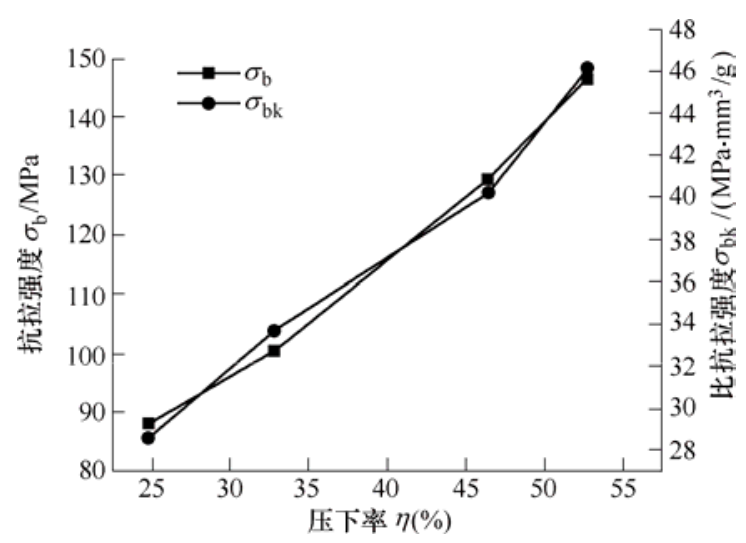

图 7 压下率 $\eta$ 对抗拉强度、比抗拉强度的影响

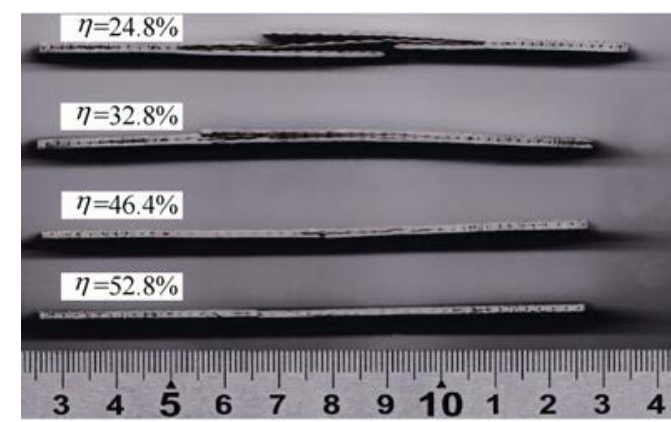

图 8 不同压下率 $\eta$ 下复合板拉伸试样拉断后侧视形貌

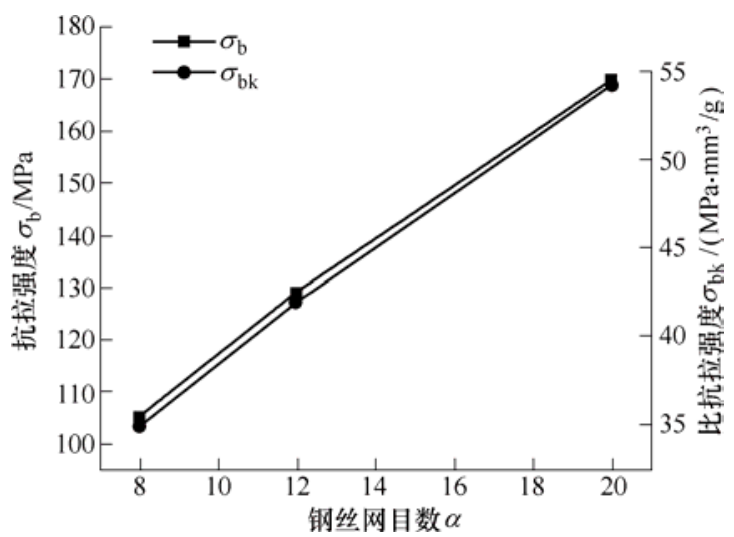

图 9 钢丝网目数 $\alpha$ 对抗拉强度、比抗拉强度的影响

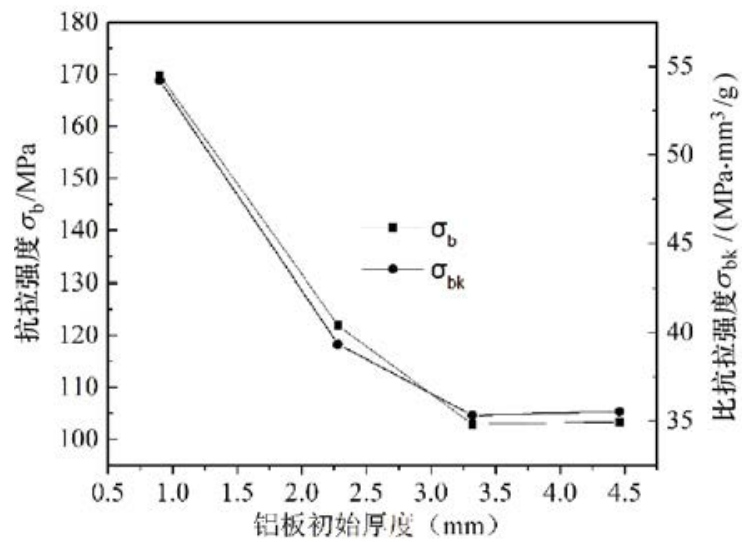

图 10 铝板初始厚度 $h$ 对抗拉强度、比抗拉强度的影响

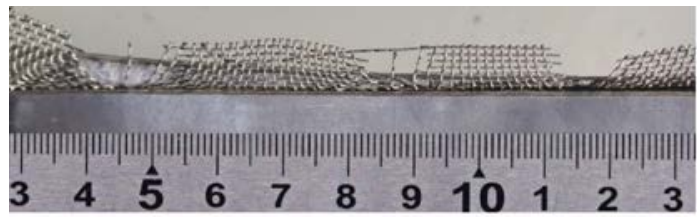

图 11 初始厚度 $3.36 \mathrm{~mm}$ 复合板形貌

（3）钢丝网摆放角度的影响。复合板拉伸力学 性能曲线如图 12 所示, 其拉伸试样拉断后宏观形貌 如图 13 所示。可以看出不同角度钢丝网增强复合板 抗拉强度都高于无钢丝网增强复合板, $45^{\circ}$ 钢丝网 增强复合板抗拉强度最大, 比 $0^{\circ}$ 钢丝网增强复合 板大 $9.5 \%$, 比无钢丝网增强复合板大 $34.2 \%$ 。不同 角度钢丝网增强复合板伸长率都低于无钢丝网增强 复合板, $45^{\circ}$ 钢丝网增强复合板伸长率高于 $0^{\circ}$ 钢 丝网增强复合板, 略低于无钢丝网增强复合板。

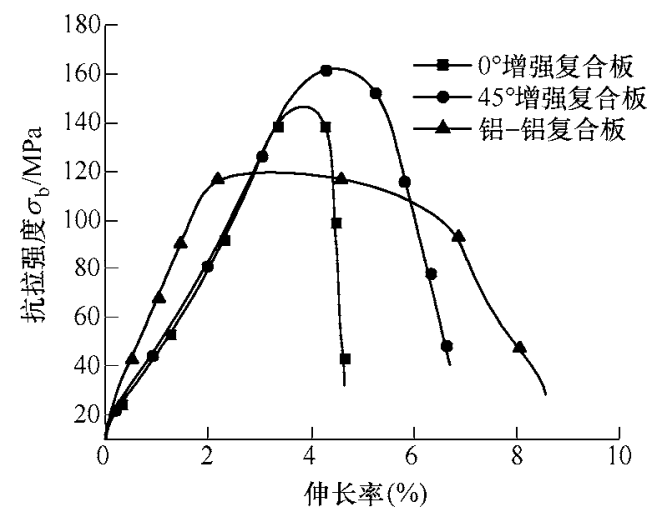

图 12 复合板拉伸力学性能曲线

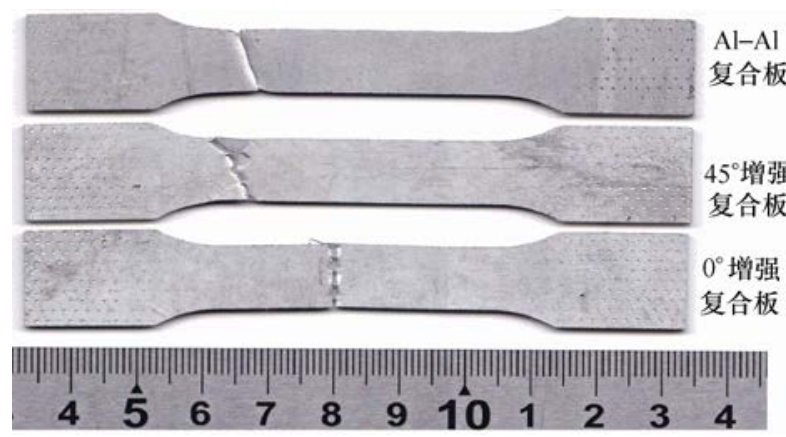

图 13 复合板拉伸试样拉断后宏观形貌 
图 14 给出了钢丝网摆放角度对其延展性的影 响机制, 可以看出, 钢丝网摆放角度为 $45^{\circ}$ 比 $0^{\circ}$ 具 有更好的延展性, 能更好地满足与基体间的变形协 调性要求。这与复合板拉伸曲线中的断裂伸长率规 律一致。

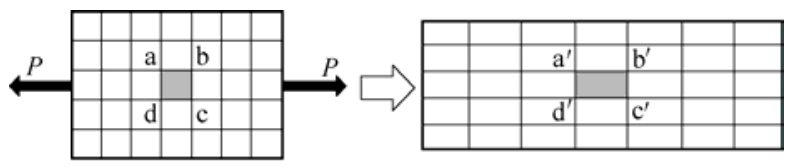

(a) $\theta=0^{\circ}$

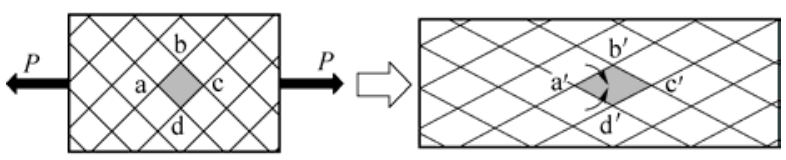

(b) $\theta=45^{\circ}$

图 14 不同角度 $\theta$ 钢丝网延展性示意图

\section{3 结论}

通过冷轧法制备了钢丝网增强铝基复合板，对 钢丝网与铝板界面结合过程进行分析, 同时探究了 不同工况下复合板的力学性能, 得到结论如下。

(1) 随着冷轧压下率的增大, 界面结合由最初 的钢丝网与铝板间机械咬合转变为局部点焊合, 而 后钢丝网与铝板结合界面间的残余孔隙、孔洞逐渐 变少, 界面结合转变为钢丝网与铝板间的面焊合。 当压下率达到 $52.8 \%$ 时, 复合界面无残留孔隙、孔 洞, 实现钢丝网与铝板间的全面焊合和铝板与铝板 间的结合。

(2) $0 \circ$ 钢丝网增强复合板抗拉强度和比抗拉强 度在一定范围内随着压下率、钢丝网目数的增大而 增大, 随着铝板初始厚度增加呈下降趋势。当铝板 初始厚度超过 $2 \mathrm{~mm}$ 后, 钢丝网增强效果较小, 同 时随着铝板初始厚度的增大, 较大压下率下钢丝网 会出现断裂, 故而采用 $0.5 \mathrm{~mm}$ 丝径的钢丝网时, 铝 板初始厚度不宜超过 $2 \mathrm{~mm}$ 。

(3) 不同角度钢丝网增强复合板抗拉强度较铝-铝 复合板都有所提高, 但伸长率有所降低。其中 $45^{\circ}$ 钢丝 网增强复合板抗拉强度提高较多, 伸长率降低较少。

\section{参 考 文 献}

[1] 刘环, 郑晓再. 层状金属复合板制备技术 [J]. 材料导 报, 2012，26(s2): 131-134.

LIU Huan, ZHENG Xiaoran. The manufacturing technique of clad metals sheet[J]. Material Review, 2012, 26(s2): 131-134.

[2] 杨益航, 王德志, 林高用, 等. 层状功能材料的研究与
发展[J]. 材料导报, 2011, 25(17): 10-13.

YANG Yihang, WANG Dezhi, LIN Gaoyong, et al. Review in research and development of laminated functional material[J]. Material Review, 2011, 25(17): 10-13.

[3] SHENG L Y, YANG F, XI T F, et al. Influence of heat treatment on interface of $\mathrm{Cu} / \mathrm{Al}$ bimetal composite fabricated by cold rolling[J]. Composites Part B Engineering, 2011, 42(6): 1468-1473.

[4] STOLBCHENKO M, GRYDIN O, SCHAPER M. Twin-roll casting and finishing treatment of thin strips of the hardening aluminum alloy EN AW-6082[J]. Materials Today Proceedings, 2015, 2: S32-S38.

[5] 沈德久, 蔡景瑞, 吴国瑞, 等. 复合轧制氧化铝-铝基 复合材料工艺与界面结合机制 [J]. 机械工程学报, 2013, 49(22): 91-96.

SHEN Dejiu, CAI Jingrui, WU Guorui, et al. Process and interface bonding mechanism of alumina reinforced aluminum matrix composite board by sandwich rolling[J]. Journal of Mechanical Engineering, 2013, 49(22): 91-96.

[6] 凌兴珠, 徐振民. 颗粒增强铝基复合材料的组织与性能 [J]. 中国有色金属学报, 1998(2): 286-290.

LING Xingzhu, XU Zhenmin. Structure and property of particle reinforced aluminium matrix composite[J]. The Chinese Journal of Nonferrous Metals, 1998(2): 286-290.

[7] 钟厉, 韩西, 周上祺. 纤维增强铝基复合材料研究进展 [J]. 机械工程材料, 2002，26(12): 12-14.

ZHONG Li, HAN Xi, ZHOU Shangqi. The progress in research of fiber-reinforced aluminium matrix composites[J]. Materials for Mechanical Engineering, 2002, 26(12): 12-14.

[8] HUANG H, LV Z, SONG S, et al. Microstructure and properties of $\mathrm{SiCp} / \mathrm{Al}$ matrix composite strip fabricating by twin-roll casting process[M]//Magnesium Technology. Springer International Publishing, 2016: 391-396.

[9] BARNES S, PASHBY I R. Machining of aluminium based metal matrix composites[J]. Applied Composite Materials, 1995, 2(1): 31-42.

[10] HAGA T, TAKAHASHI K. Casting of composite strip using a twin roll caster[J]. Journal of Materials Processing Technology, 2004, s157-158(4): 701-705.

[11] HAGA T, TAKAHASHI K, WATARI H, et al. Casting of wire-inserted composite aluminum alloy strip using a twin roll caster[J]. Journal of Materials Processing Technology, 2007, s192-193(11): 108-113.

[12] 谢建新, 刘静安. 金属挤压理论与技术 [M]. 2 版. 北京: 冶金工业出版社，2012.

XIE Jianxin, LIU Jingan. Theory and technology for metal extrusion[M]. 2nd ed. Beijing: Metallurgical Industry Press, 2012.

(下转第 137 页) 
technology, 2008, 202(1): 275-282.

[8] WEIBENGA J H, WEISS M, ROLFE B, et al. Product defect compensation by robust optimization of a cold roll forming process[J]. Journal of Materials Processing Technology, 2013(213): 978-986.

[9] JOHN P, KONSTANTINOS S , GEORGE C. Investigation of the effect of roll forming pass design on main redundant deformations on profiles from AHSS[J]. International Journal of Advanced Manufacturing Technology, 2011(56): 475-491

[10] SAFDARIAN R, NAEINI H M. The effect of forming parameters on the cold roll forming of channel section[J]. Thin-Walled Structures，2015，92: 130-136.

[11] 张宝, 丁武学, 彭斌涁. 下山法成形在辊弯成形中的应 用 $[\mathrm{J}]$. 锻压技术，2014，39(1)：52-57.
ZHANG Bao, DING Wuxue, PENG Binbin. Application of downhill method in roll forming[J]. Forging \& Stamping Technology, 2014, 39(1): 52-57.

[12] BIDABADI B S, NAEINI H M, TAFTI R A, et al. Experimental investigation of the ovality of holes on pre-notched channel products in the cold roll forming process[J]. Journal of Materials Processing Technology, 2015(225): 213-220.

[13] BIDABADI B S, NAEINI H M, TAFTI R A, et al. Experimental and numerical study of bowing defects in cold roll-formed, U-channel sections[J]. Journal of Constructional Steel Research, 2016(118): 243-253

作者简介(通信作者): 韩飞, 男, 1977 年出生, 硕士研究生导师。主要 研究方向为辊弯成形工艺及设备。

E-mail: hanfei@ncut.edu.cn

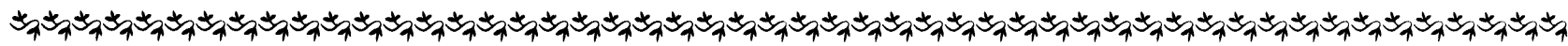

\section{(上接第 130 页)}

[13] PAKZAMAN H R, DIVANDARI M, KHAVANDI A R. Effect of nickel coating on steel wire reinforcement on mechanical properties of aluminum matrix composites produced via lost foam casting[C/CD]//Iran International Aluminum Conference, 2012.

[14] GÜLENÇ B, KAYA Y, DURGUTLU A, et al. Production of wire reinforced composite materials through explosive welding[J]. Archives of Civil \& Mechanical Engineering, 2016, 16(1): 1-8.

[15] SZCZEPANIK S. Composites of aluminium alloy matrix reinforced by a steel mesh[J]. Materials Today
Proceedings, 2015, 2: 9-18.

作者简介: 黄华贵(通信作者), 男, 1978 年出生, 博士, 教授, 博士研 究生导师。主要研究方向为层状金属复合材料制备工艺及装备、数值模 拟技术及工程应用。

E-mail: hhg@ysu.edu.cn

王计超, 男, 1993 年出生, 硕士研究生。主要研究方向为不锈钢丝网增 强铝基复合板轧制复合技术。

E-mail:2567214873@qq.com

刘文文, 男, 1990 年出生, 博士研究生。主要研究方向为层状金属复合 材料固-液铸轧复合技术。

E-mail: 837725878@qq.com

杜风山, 男, 1960 年出生, 教授, 博士研究生导师。主要研究方向为双 辊振动铸轧近终成形工艺与装备技术、轧机数字化技术。

E-mail: fsdu@ysu.edu.cn 